\section{Osteomyelitis of Metatarsal Sesamoid}

The metatarsal sesamoids are rarely the site of pathological processes, which are common in other bones. Pyogenic infection of these bones is especially unusual and diagnosis is often difficult and delayed.

\section{CASe History}

A young man was admitted to hospital complaining of severe pain and swelling of the metatarso-phalangeal joint of the right great toe. The pain had begun suddenly three days prior to admission and had been regarded by his family doctor as acute gout. Three days' treatment with colchicine had failed to give relief. There was no history of injury to the foot.

On examination the affected joint and the surrounding tissues were swollen, slightly hot, and extremely tender. His temperature was $99.4^{\circ} \mathrm{F}$. $\left(37.4^{\circ} \mathrm{C}\right.$.). No abnormality was detected in other systems on general examination. The white-cell count showed leucocytosis. The erythrocyte sedimentation rate was $7 \mathrm{~mm}$. in one hour (Westergren). Repeated estimations of serum uric acid concentration were normal. Blood culture was sterile after 10 days' incubation. Radiographs of the foot (Fig. 1) on the day of admission showed no abnormality. A provisional diagnosis of acute pyogenic infection of the subcutaneous tissue of the region of the first metatarso-phalangeal joint was made. He was treated by bed rest and systemic penicillin. After two weeks he developed a blister on the medial aspect of the joint, which contained thin pus. Pyogenic staphylococci sensitive to penicillin were grown from the contents of this blister. Further radiographs (Fig. 2) at this time showed slight separation of the medial from the lateral sesamoid bone. A diagnosis of pyogenic osteomyelitis of the medial sesamoid was then made and antibiotic therapy continued. Two discharging sinuses developed in the depths of the blister.

Radiographs (Fig. 3) taken 38 days after onset of symptoms showed that the smaller fragment of the medial sesamoid had separated from its fellow and was lying beneath the skin of the medial aspect of the metatarso-phalangeal joint. The other part of the medial sesamoid has also moved proximo-laterally from its original position. The lateral sesamoid bone appeared normal. Removal of the sesamoid bone was followed by complete recovery. The medial sesamoid bone had the appearance of bony sequestra.

\section{COMMEnT}

As the radiographic changes are extremely important in the diagnosis of this condition the normal anatomy should be understood. The metatarsal sesamoid bones in the tendons of flexor hallucis brevis are constantly present. Ossification begins at the age of 8 to 10 years (Inge and Ferguson, 1933). Normally there may be one, two, or more centres of ossification, and these multiple centres may or may not fuse, giving rise to solitary, bipartite, or multipartite sesamoid consisting of unequal fragments. In 6 to $8 \%$ of feet there is division of these bones. Of these, the medial sesamoid is affected in $90 \%$ (Bennet, 1935). The reason for this division and the disparity of division is unknown.

In the antero-posterior view the medial sesamoid is larger than the lateral and is situated further forward. It usually lies within the outline of the head of the first metatarsal, whereas the lateral sesamoid has a tendency to extend beyond the lateral boundary of the head of this bone. In the lateral view the sesamoids lie opposite the articular facets on the plantar aspect of the head of the first metatarsal.

In the above case the radiographic appearances in the initial stage were within normal limits. In early osteomyelitis this is a common finding. Further radiographs taken after 17 days showed slight separation of the fragments of medial sesamoid, and later radiographs showed their extrusion from their original site.

The delay in the radiographic changes can be explained. At first, local infection takes the form of a small intraosseous abscess. This is not obvious in the early radiographs. Because of the small size of the bone, this abscess breaks into the subperiosteal region. It extends rapidly beneath the periosteum, isolates the sesamoid bone from its blood supply, and leads to the formation of a sequestrum. Only then can one notice the changes in texture of the bone and the alteration in its position.

In the cases recorded by Lange (1928), Bennet (1935), Smith (1941), and Frank (1943) there was difficulty and delay in diagnosis. In all cases the acute stage was followed after a long interval by blister and abscess formation. Eventually fistulae developed and the sesamoids became sequestra. There was also delay in radiographic changes.

Schütz (1928) believed that only in the presence of division of the sesamoids could pathological conditions occur in the sesamoids. It is understandable that pressure on the bipartite sesamoid while standing could

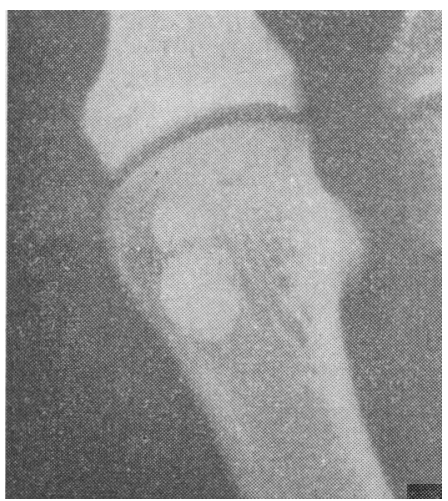

FiG. 1.-Radiograph taken three days after onset of symptoms. Medial metatarsal sesamoid is bipartite.

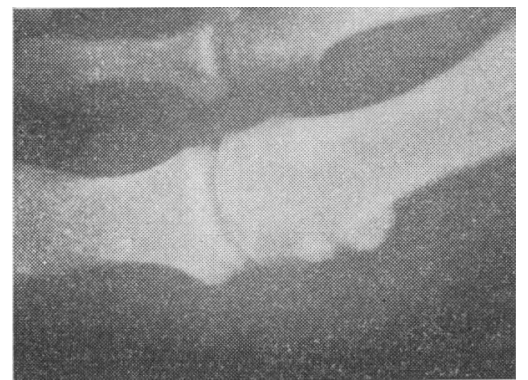

FIG. 2.-Radiograph taken 20 days after onset of symptoms. There is slight increase in gap between the two fragments of the bipartite metatarsal sesamoid Density of this bone has increased.

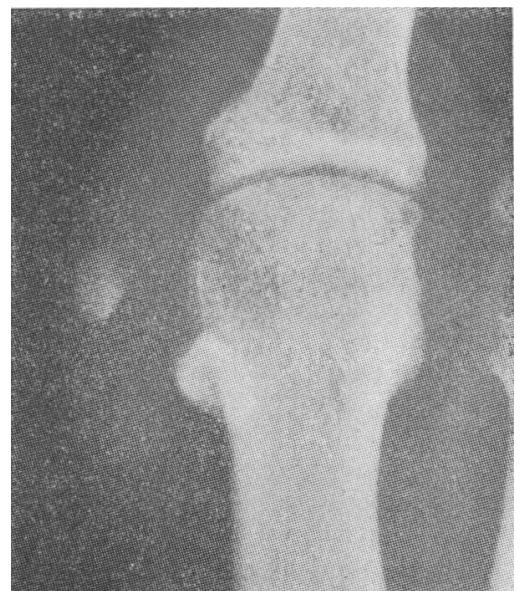

FIG. 3.-Radiograph taken 38 days after onset of symptoms. Fragments are displaced considerably and show increased density. 
create a distracting force on the fragments. This could easily give rise to a local haematoma. Hopps (1953) suggested that frequent bacteriaemia can take place without any symptoms. In the present case, therefore, local haematoma followed by haematogenous infection could be the mode of origin of osteomyelitis. The delay in operation does not appear to cause any harm, as excision of the diseased bone was followed by complete cure in all the five cases recorded. This can be explained by the localized nature of the lesion. Removal of the sesamoid gives a permanent cure.

My thanks are extended to Mr. E. Mervyn Evans, under whose care the patient was admitted.

M. M. Rowe, F.R.C.S.,

Senior Registrar in Orthopaedic and Traumatic Surgery, Morriston Hospital, Swansea.

\section{REFERENCES}

Bennet, K. (1935). Acta chir. scand., 76, 103.

Frank, T. J. F. (1943). Roy. Melb. Hosp. clin. Rep., 14, 80 Hopps, H. C. (1953). Pathology, edited by W. A. D. Anderson, 2nd ed., p. 199. Kimpton, London

Inge, G. A. L., and Ferguson, A. B. (1933). Arch. Surg., 27. 466.

Lange, M. (1928). Orthop. Chir., 49, 595

Schütz, H. (1928). Beitr. Z klin. Chir., 145, 65.

Smith, R. (1941). Brit. J. Surg., 29, 19.

\section{Allergic Pulmonary Infiltration Due to Nitrofurantoin}

An increasing number of drugs are known to be associated with allergic reactions manifested by eosinophilia and transient pulmonary infiltration of the type originally described by Löffler (1932). A case due to nitrofurantoin ("furadantin") has recently been described (Israel and Diamond, 1962). We now record a second example of this association.

\section{CASE RePort}

The patient, a 54-year-old physician, had previously suffered from hay-fever and allergy to sticking-plaster but not from asthma. In 1957 he suffered an attack of cystitis, for which he received nitrofurantoin $100 \mathrm{mg}$. six-hourly without ill effect.

In August, 1962, he developed a further attack of cystitis and received nitrofurantoin four-hourly for four days. On the evening of the fifth day he developed a fever with shivering, a dry cough, and slight shortness of breath. The next morning his own doctor noted rales at both bases but no bronchospasm. He was treated with penicillin and streptomycin, and improved rapidly. Two weeks later his cystitis recurred and again nitrofurantoin was given. One hour after taking the second dose of $100 \mathrm{mg}$. he rapidly developed symptoms similar to those of the previous episode and consisting of shivering, fever, difficulty with breathing, and a cough with a small amount of whitish sputum. $\mathrm{He}$ was admitted to hospital the same afternoon and on examination his temperature was $101^{\circ} \mathrm{F} .\left(38.3^{\circ} \mathrm{C}\right.$.). At both bases loud rales could be heard as high as the angle of the scapula. There was no bronchospasm, cyanosis, or pleuritic pain, but he appeared to have difficulty in breathing.

He was initially treated with tetracycline, but by the following morning the whole episode appeared to have abated, the temperature being normal and the lungs practically clear on auscultation.

Investigations at this time showed: haemoglobin $12.9 \mathrm{~g} . /$ $100 \mathrm{ml}$.; leucocytes $6,800 / \mathrm{c} . \mathrm{mm}$. (neutrophils $60 \%$, lymphocytes $16 \%$, monocytes $4 \%$, eosinophils $20 \%$ ). The chest radiograph (see Fig.) showed diffuse mottling in both lower zones.
The tetracycline was continued for five days, as we were not aware of the association of Löffler's syndrome with nitrofurantoin. His recovery was virtually complete on the second day after admission and a chest radiograph on the fifth day was normal. The eosinophilia also subsided to $2 \%$ of a total leucocyte count of $11,000 / \mathrm{c} . \mathrm{mm}$. No pathogens were isolated from the sputum, and viral antibody studies showed no evidence of recent infection.

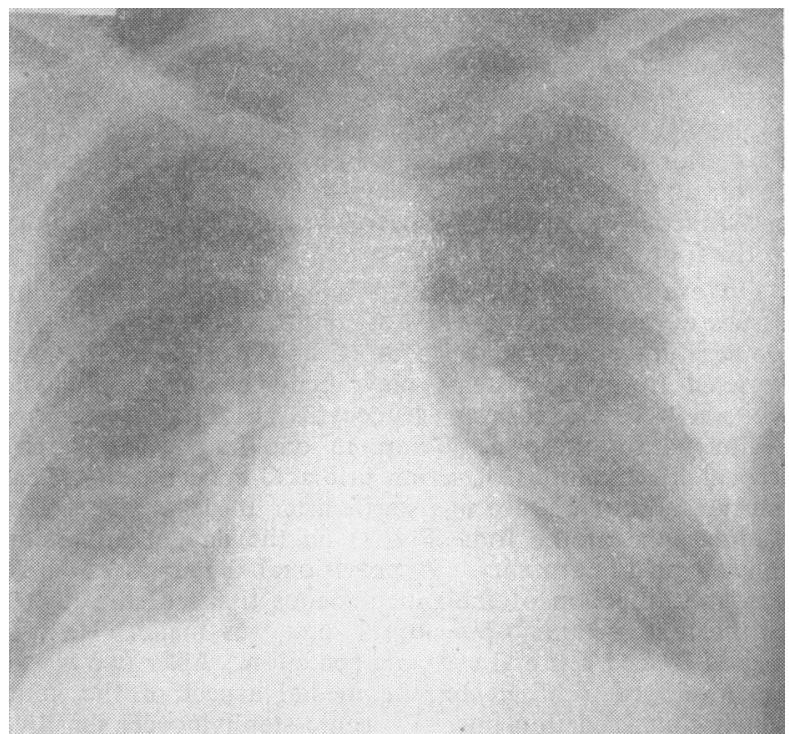

Radiograph showing diffuse mottling in both lower zones.

He was discharged with instructions to avoid contact with nitrofurantoin. The cause of the recurrent cystitis is under investigation.

\section{COMMENT}

Israel and Diamond were able to prove that nitrofurantoin was the causative factor in their case by reproducing the illness with a small dose of the drug. We are not able to do this, as the patient was anxious to return to his medical practice without delay. There can be little doubt about the cause of his illness, however, which was clearly allergic in origin and closely followed nitrofurantoin on each occasion. The increasing speed of onset with each episode noted by Israel and Diamond was also apparent.

Reactions to nitrofurantoin are not common, as the drug is widely prescribed and reports of allergy are few. Circulatory collapse occurred on one occasion (Fisk, 1957).

Other drugs known to cause pulmonary eosinophilia include p-aminosalicylic acid (Waring and Howlett, 1952), penicillin (Reichlin et al., 1953), and mephenesin (Rodman et al., 1958).

We wish to thank Dr. F. H. Scadding for permission to publish this case.

D. C. F. MuIR, M.B., B.S., M.R.C.P.

J. A. Stanton, M.B., B.S.

\section{Middlesex Hospital,}

London.

\section{RBFERENCES}

Fisk, A. A. (1957). New Engl. J. Med., 256, 1054

Israel $H$ L and Diamond P (1962), Ibid 266, 1024

isracl,

Reichlin S Loveless, M. H. and Kane, E. G. (1953), Ann. intern. Med., 38, 113 .

Rodman, T., Fraimow, W., and Myerson, R. M. (1958). Ibid. 43, 668 .

Warring, F. C., and Howlett, K. S. (1952). Amer. Rev. Tuberc. 65,235 